\title{
Aneurismas associados à fenestração da artéria basilar: entidades raras submetidas a tratamento endovascular - Relato de casos e revisão da literatura
}

\author{
Johnathan de Sousa Parreira', Adriano Torres Antonucci², \\ Natally Marques Santiago ${ }^{2}$, Roberto Parente Neto², Bruno de Azevedo Oliveira ${ }^{3}$, \\ Luiz Henrique Garcia Lopes ${ }^{3}$, Adelmo Ferreira ${ }^{3}$, Pedro Garcia Lopes ${ }^{4}$ \\ Hospital Universitário Regional do Norte do Paraná, Universidade Estadual de Londrina, Londrina, PR, Brasil.
}

\section{RESUMO}

Aneurismas da junção vertebrobasilar são raros e frequentemente associados com fenestração da artéria basilar. Estudos mostram que tal entidade representa entre $3 \%$ e $9 \%$ dos aneurismas infratentoriais e $7,4 \%$ dos aneurismas da circulação posterior, excluindo-se os aneurismas da bifurcação da basilar. A sua localização profunda, anterior ao tronco cerebral, e sua intima relação com as artérias perfurantes tornam o tratamento cirúrgico um grande desafio. A fenestração foi mais comumente encontrada na metade inferior da basilar, situando-se na porção média quando as artérias vertebrais apresentam as mesmas dimensões ou encontradas do lado correspondente à artéria vertebral de maior calibre. Outras classificações para a fenestração da basilar foram propostas. De acordo com a mais utilizada, a fenestração pode ser considerada do tipo $A$ quando apresentar dois pontos de bifurcação proximais, com uma artéria ponte associada e do tipo $B$ quando apresentar bifurcação simples proximal ao sítio da fenestração. Tais aneurismas são de difícil caracterização pela angiografia digital convencional por apresentarem anatomia peculiar, sendo a angiografia em 3D o exame de escolha para a classificação da doença. As modernas técnicas endovasculares disponíveis na atualidade permitem o tratamento seguro mesmo de lesões altamente complexas.

\section{PALAVRAS-CHAVE}

Aneurisma intracraniano, angiografia cerebral, artéria basilar, artéria vertebral.

\section{ABSTRACT}

Aneurysms at fenestration basilar artery: rare entities submitted to endovascular treatment Case report and literature review

Aneurysms of the vertebrobasilar junction are rare and often associated with fenestration of the basilar artery. Studies show that such entity represents between 3\% and 9\% of infratentorial aneurysms and 7.4\% of aneurysms of the posterior circulation excluding basilar bifurcation aneurysms. Its location deep, anterior to the brainstem and its intimate relationship with the perforating arteries, becomes surgical treatment a challenge. The fenestration was more commonly found in the lower half of the basilar, standing in the middle portion when the vertebral arteries have the same dimensions and found the corresponding side of the vertebral artery of larger caliber. Other ratings for fenestration of the basilar were proposed. According to these most used, the fenestration can be regarded as type $A$, when present two bifurcation points with a proximal artery associated bridge, and the type $B$ when presenting simple bifurcation proximal to the site of fenestration. Such aneurysms are difficult to characterize by conventional digital angiography for presenting peculiar anatomy, 3D angiography is the exam of choice for disease classification. Modern endovascular techniques currently available allow for the safe treatment of even highly complex lesions.

\section{KEYWORDS}

Intracranial aneurysm, cerebral angiography, basilar artery, vertebral artery.

1 Neurocirurgião e ex-residente do Serviço de Neurocirurgia do Hospital Universitário Regional do Norte do Paraná da Universidade Estadual de Londrina (UEL), Londrina, PR, Brasil.

2 Residente do Serviço de Neurocirurgia do Hospital Universitário Regional do Norte do Paraná da UEL, Londrina, PR, Brasil.

3 Neurocirurgião e docente do Serviço de Neurocirurgia do Hospital Universitário Regional do Norte do Paraná da UEL, Londrina, PR, Brasil.

4 Neurocirurgião e chefe do Serviço de Neurocirurgia do Hospital Universitário Regional do Norte do Paraná da UEL, Londrina, PR, Brasil. 


\section{Introdução}

Os aneurismas saculares da junção vertebrobasilar são raros, mas, quando presentes, frequentemente estão associados à fenestração da artéria basilar. ${ }^{1} \mathrm{~A}$ artéria basilar é o segundo local mais comum de fenestrações de artérias intracranianas, depois da artéria vertebral. ${ }^{2,3}$ Fenestração da artéria basilar está presente em $0,6 \%$ das angiografias e aproximadamente $5 \%$ de algumas séries de autópsias. ${ }^{4,5}$ Ela pode ocorrer em qualquer lugar ao longo da basilar, mas é mais comum próximo à junção vertebrobasilar. A anatomia complexa da região, com múltiplos ramos perfurantes para o tronco cerebral, além da proximidade com os nervos cranianos, torna a clipagem cirúrgica difícil. Recentemente, para esses casos, sugere-se o tratamento endovascular. No presente trabalho, descrevemos dois casos de aneurismas associados à fenestração da artéria basilar, tratados em único centro de referência, admitidos em um curto espaço de tempo (um mês). O Hospital Universitário Regional do Norte do Paraná, anexo à Universidade Estadual de Londrina, é reconhecido, na atualidade, como único centro de referência, pelo Sistema Único de Saúde (SUS), para tratamento endovascular gratuito de aneurismas cerebrais fora da capital do estado.

\section{Relato dos casos}

Paciente 1: 43 anos, sexo feminino, com quadro de cefaleia holocraniana intensa, de início súbito, associado à rigidez de nuca, com melhora espontânea há oito dias. Houve recidiva do quadro, acrescido de desmaio, quando a paciente foi atendida em hospital da sua cidade, submetida a exames complementares e encaminhada para o nosso serviço. No exame físico, encontrava-se consciente e orientada, sem qualquer outra alteração no exame neurológico. Trouxe consigo tomografia de crânio (TC), com contraste, que mostrou imagem arredondada junto à artéria basilar (Figura 1); uma ressonância magnética de crânio (RM) e angiorressonância que mostrou obliteração dos sulcos na sequência FLAIR sugerindo discreta hemorragia subaracnóidea; discreta ectasia do sistema ventricular e imagem sugestiva de formação aneurismática no segmento proximal da artéria basilar (Figura 2). A paciente foi submetida à angiografia cerebral, com estudo por ambas as artérias vertebrais, sendo detectada fenestração na origem da artéria basilar com aneurisma irregular em seu segmento comunicante, medindo aproximadamente $8 \mathrm{~mm}$ na sua maior extensão, de colo estreito (Figuras 3 e 4). Diante do diagnóstico, realizou-se embolização do aneurisma com micromolas destacáveis, sob anestesia geral, sem intercorrências (Figura 5), sendo a paciente encami- nhada para observação na unidade de terapia intensiva, recebendo alta em 24 horas sem qualquer alteração no exame neurológico. A paciente recebeu alta hospitalar em três dias, após o tratamento endovascular.

Paciente 2: 54 anos, branca, encaminhada de outro serviço e admitida no pronto-socorro com quadro de confusão mental, cefaleia e vômitos há 6 horas. Apresentava, ainda, sonolência e rigidez de nuca. Trouxe consigo angiografia cerebral com aneurisma na junção vertebrobasilar, associado à fenestração da artéria basilar (Figuras 6 e 7). Diante do diagnóstico, a paciente foi encaminhada para a sala de hemodinâmica e submetida a tratamento endovascular, sendo realizada a embolização do aneurisma com micromolas destacáveis (Figuras 8 e 9), sob anestesia geral, sem intercorrências. Após o procedimento, a paciente foi encaminhada para a unidade de terapia intensiva, de onde recebeu alta após 24 horas sem qualquer alteração no exame neurológico. A paciente recebeu alta hospitalar após 72 horas do tratamento endovascular.

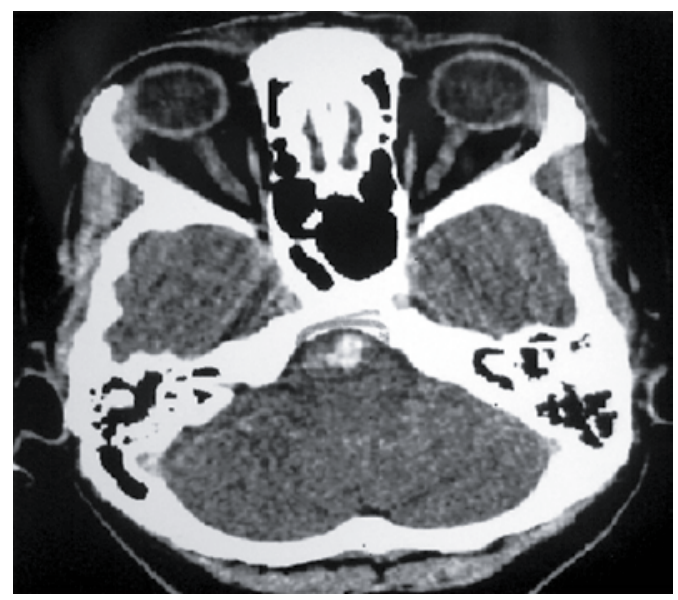

Figura 1 - Paciente 1: Tomografia de crânio, corte axial, com imagem em topografia da artéria basilar, sugestiva de aneurisma cerebral.

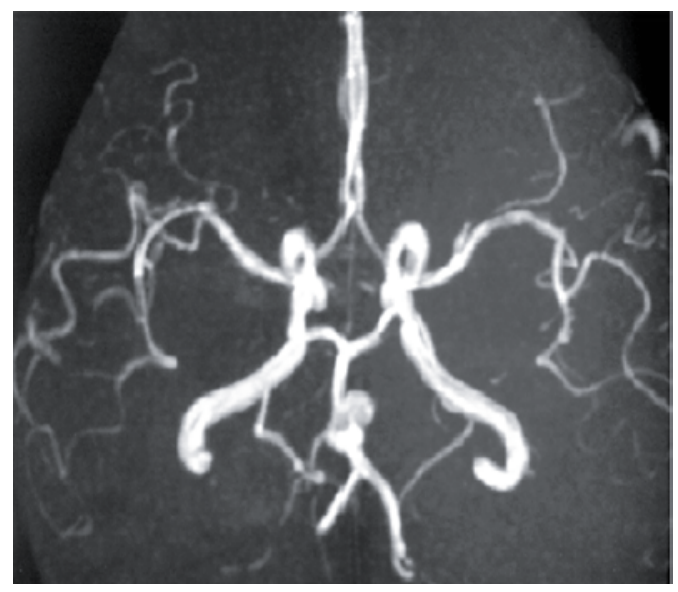

Figura 2 - Paciente 1: Angiorressonância cerebral demonstrando aneurisma na junção vertebrobasilar. 


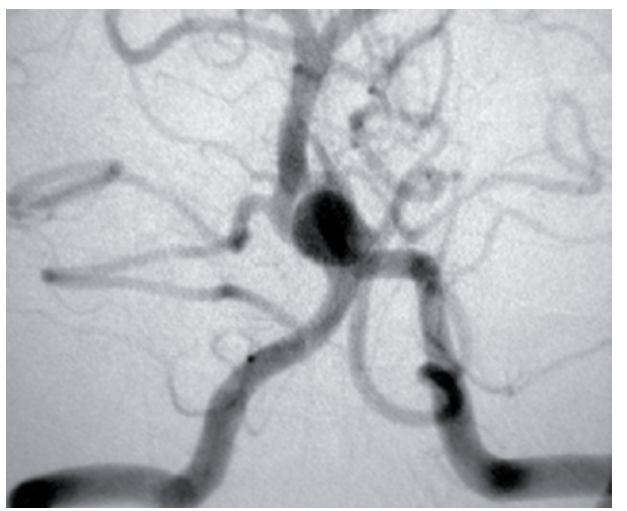

Figura 3 - Paciente 1: Angiografia cerebral, em incidência anteroposterior, demonstrando aneurisma, na junção vertebrobasilar, associado à fenestração da artéria basilar.

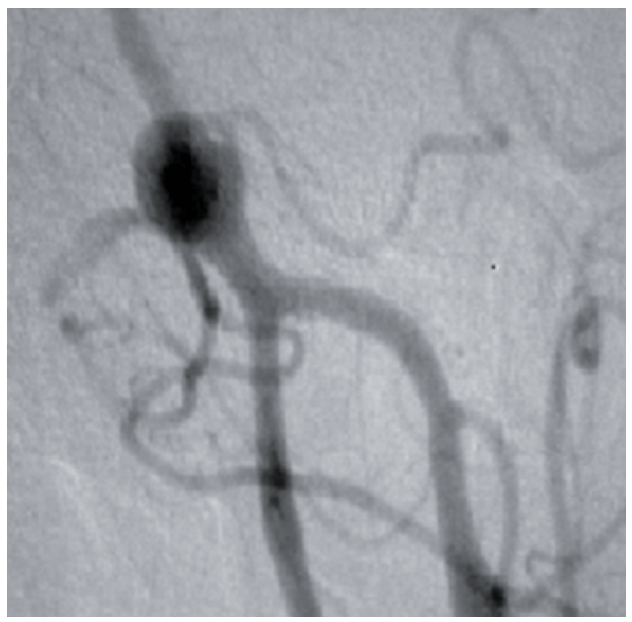

Figura 4 - Paciente 1: Angiografia cerebral, em incidência oblíqua, demonstrando aneurisma associado à fenestração da artéria basilar.

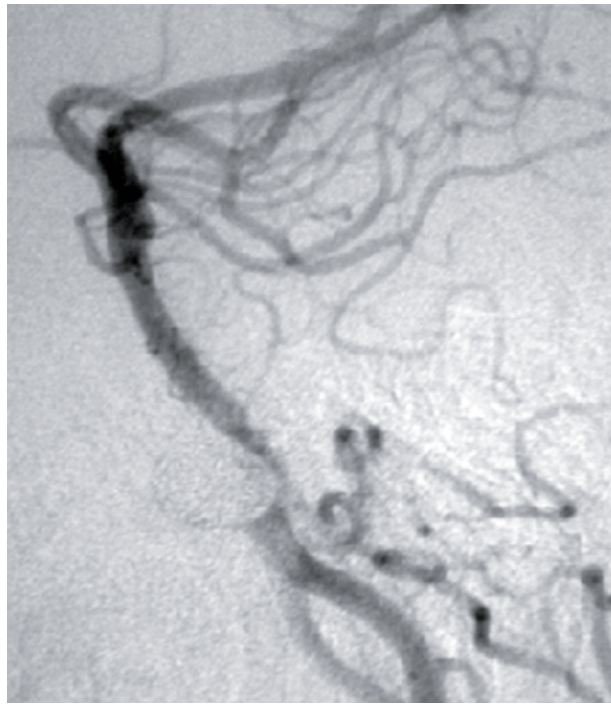

Figura 5 - Paciente 1: Angiografia cerebral mostrando aneurisma embolizado com espiras destacáveis, sendo mantida a circulação nos ramos arteriais adjacentes.

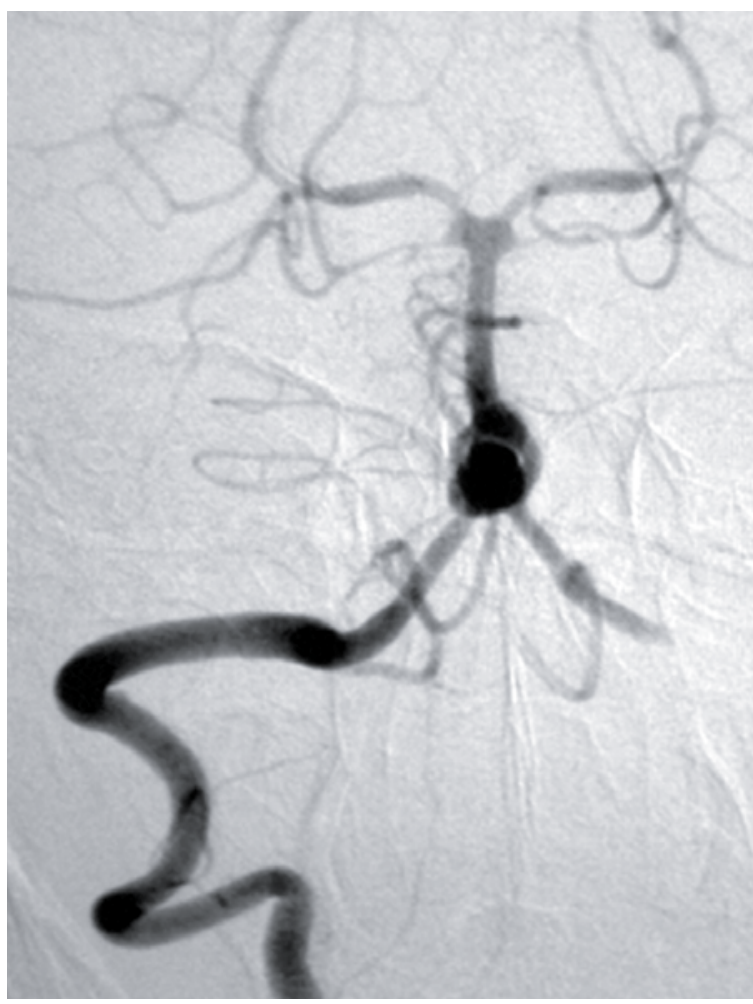

Figura 6 - Paciente 2: Angiografia cerebral, na incidência anteroposterior, com aneurisma na junção vertebrobasilar associado à fenestração da artéria basilar.

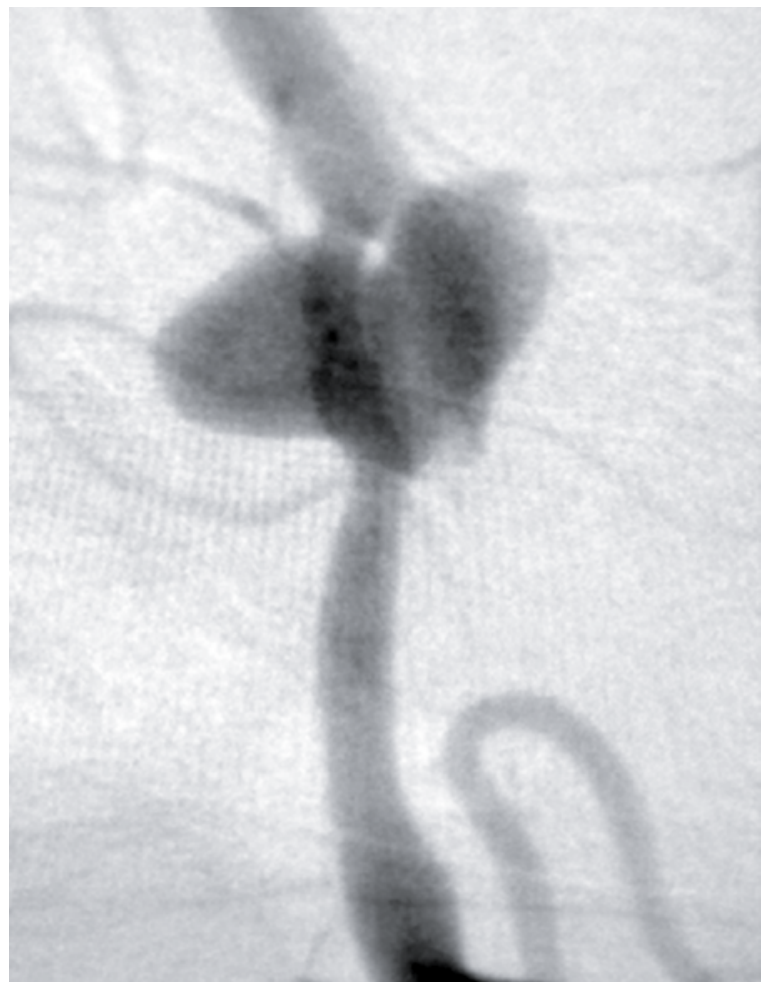

Figura 7 - Paciente 2: Angiografia cerebral, incidência lateral, destacando o aneurisma e a fenestração da artéria basilar. 


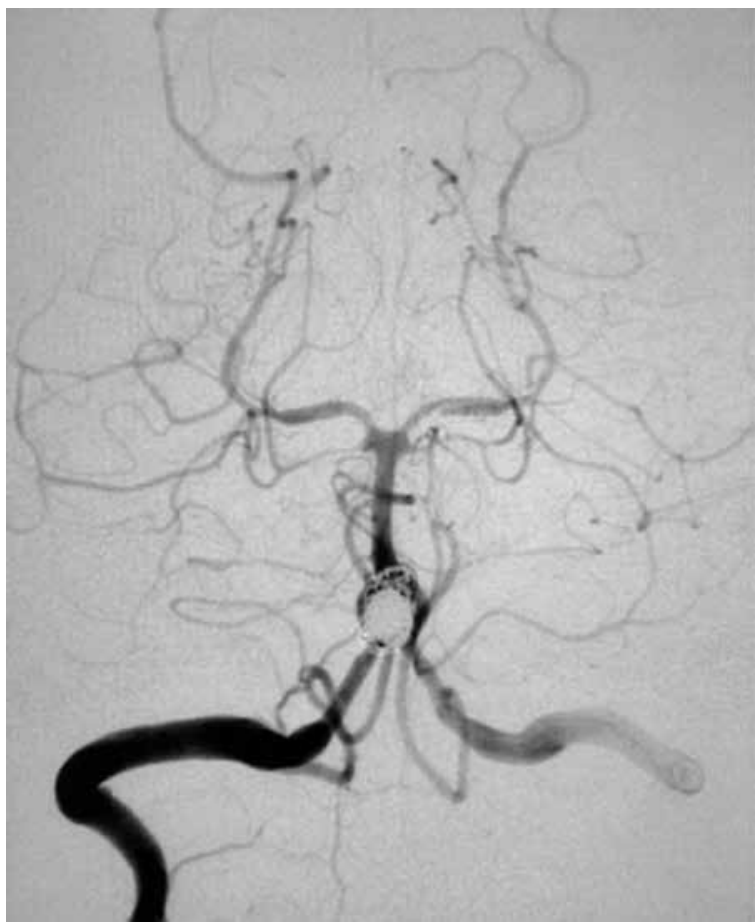

Figura 8 - Paciente 2: Angiografia cerebral, incidência anteroposterior, mostrando aneurisma embolizado com espiras destacáveis, com manutenção do fluxo sanguíneo nos segmentos arteriais adjacentes.

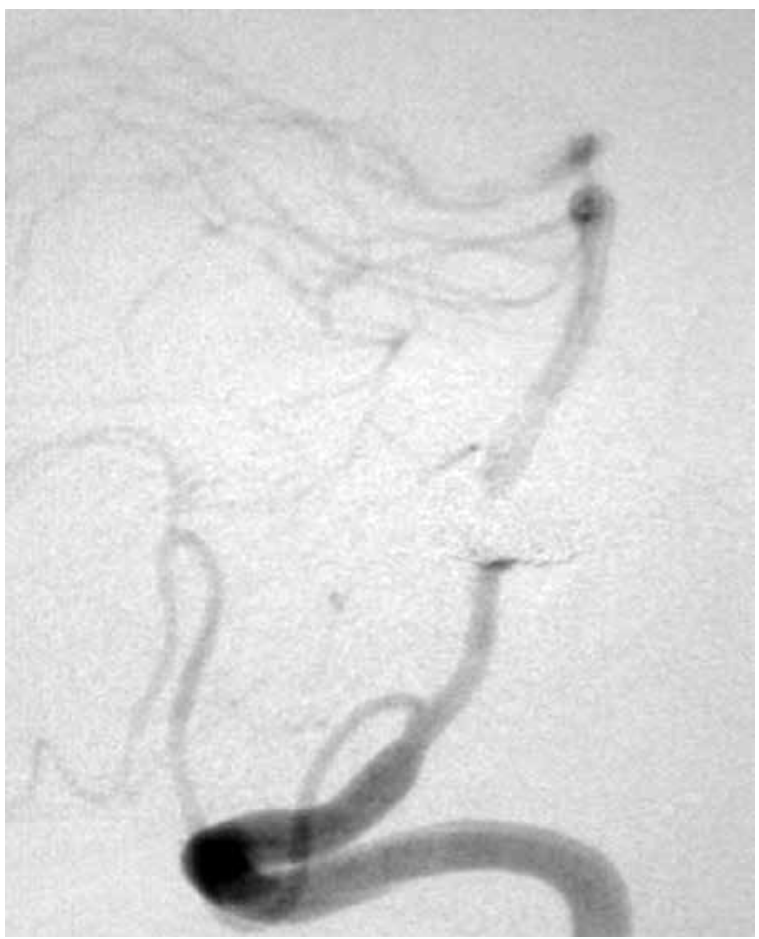

Figura 9 - Paciente 2: Angiografia cerebral, incidência lateral, mostrando aneurisma embolizado com espiras destacáveis, com manutenção do fluxo sanguíneo nos segmentos arteriais adjacentes.

\section{Discussão}

A artéria basilar é formada pela fusão de artérias neurais longitudinais paralelas durante a quinta semana de gestação. Durante esse processo de fusão, existem artérias pontes temporárias conectando as duas artérias longitudinais, que regridem quando a fusão está completa. Se houver persistência de uma das artérias pontes, ocorre a fenestração da artéria basilar. ${ }^{5,6}$ A parede lateral da artéria fenestrada tem arquitetura normal, entretanto a parede medial mostra septações, dividindo o lúmen vascular em dois canais distintos, apresentando defeitos focais em ambas as extremidades da fenestração, o que predispõe à formação de aneurismas. ${ }^{7}$ As fenestrações arteriais intracranianas podem ocorrer tanto na circulação anterior (artéria cerebral anterior e artéria cerebral média) quanto na circulação vertebrobasilar, sendo mais comuns nesta.

A maioria dos aneurismas associados à fenestração ocorre no tronco proximal à junção vertebrobasilar. Campos et al. ${ }^{8}$ apresentaram 21 casos de aneurismas associados à fenestração da artéria basilar, todos proximais à junção vertebrobasilar. Em nossos casos, ambos encontravam-se nessa mesma localidade. A incidência de aneurisma na presença de fenestração corresponde a 7\%. ${ }^{6}$ A incidência de fenestração, quando detectado um aneurisma na junção vertebrobasilar, corresponde a $35,5 \%{ }^{8}$ Assim sendo, devemos suspeitar de fenestração quando nos deparamos com aneurisma nesse local. Para melhor estudo da fenestração, torna-se necessária a angiografia em $3 \mathrm{D}$, de que não dispomos em nossa instituição. Além disso, recomenda-se o estudo angiográfico de ambas as vertebrais. Alguns trabalhos ressaltam a possibilidade de uso da angiotomografia, com excelentes resultados. ${ }^{9}$ A fenestração pode ser classificada, de acordo com seu tamanho, ${ }^{9} \mathrm{em}$ pequena (0-3 mm), média (3,1-5 mm) e grande (> 5,1 mm). Kai et al. ${ }^{10}$ propuseram uma classificação diferente, segundo a qual a fenestração pode ser considerada do tipo A, quando apresentar dois pontos de bifurcação proximais com uma artéria ponte associada, e do tipo $\mathrm{B}$, quando apresentar bifurcação simples proximal ao sítio da fenestração.

O acesso cirúrgico à junção vertebrobasilar é prejudicado pelo osso petroso. Além disso, a proximidade do aneurisma com o tronco cerebral, com inúmeras artérias perfurantes emergindo da artéria basilar, e a presença dos nervos cranianos dificultam a clipagem, mesmo com as modernas técnicas microcirúrgicas. Diante dessas dificuldades, o tratamento endovascular tornou-se uma alternativa viável para o tratamento seguro e eficiente desse tipo de aneurisma, com excelentes resultados a pequeno e médio prazo. ${ }^{1,11-13} \mathrm{Em}$ nosso trabalho, os dois aneurismas foram embolizados com molas destacáveis, sendo totalmente excluídos da 
circulação, sem intercorrências, com manutenção do fluxo nos ramos perfurantes proximais.

\section{Conclusão}

Aneurismas da junção vertebrobasilar são raros, com incidência de $0,5 \%$ de aneurismas tratados. A maioria dos aneurismas localizados no tronco proximal à junção vertebrobasilar está associada à fenestração. A angiografia em 3D com o estudo das duas vertebrais continua sendo o exame de escolha para estudo e classificação da lesão. A angiotomografia pode ser um exame valioso nos casos em que a angiografia não está disponível. O tratamento endovascular é eficaz e seguro na exclusão dos aneurismas da circulação.

\section{Referências}

1. Bavinzski G, Killer M, Gruber A, Reinprecht A, Gross CE, Richling $B$. Treatment of basilar artery bifurcation aneurysms by using Guglielmi detachable coils: a 6-year experience. J Neurosurg. 1999;90(5):843-52.

2. Osborn RE, Kirk G. Cerebral arterial fenestration. Comput Radiol. 1987;11(3):141-5.

3. Picard L, Roy D, Bracard S, Per A, Marchal JC. Aneurysm associated with a fenestrated basilar artery: report of two cases treated by endovascular detachable balloon embolization. AJNR Am J Neuroradiol. 1993;14(3):591-4.

4. Takahashi M, Tamakawa Y, Kishikawa T, Kowada M. Fenestration of the basilar artery. Report of three cases and review of the literature. Radiology. 1973;109(1):79-82.
5. Giuffrè R, Sherkat S. The vertebral artery: developmental pathology. J Neurosurg Sci. 1999;43(3):175-89.

6. Sanders WP, Sorek PA, Mehta BA. Fenestration of intracranial arteries with special attention to associated aneurysms and other anomalies. AJNR Am J Neuroradiol. 1993;14(3):675-80.

7. Finlay HM, Canham PB. The layered fabric of cerebral artery fenestrations. Stroke. 1994;25(9):1799-806.

8. Campos J, Fox AJ, Viñuela F, Lylyk P, Ferguson GG, Drake $C G$, et al. Saccular aneurysms in basilar artery fenestration. AJNR Am J Neuroradiol. 1987;8(2):233-6.

9. Foroni LH, Figueiredo EG, Teixeira MJ, Caldas JG, Leszczynski A, Rivau FR. Saccular aneurysms at middle basilar trunk fenestration. Arq Neuropsiquiatr. 2010;68(2):309-11.

10. Kai Y, Hamada J, Morioka M, Yano S, Fujioka S, Kuratsu J. Endovascular treatment of ruptured aneurysms associated with fenestrated basilar artery. Two case reports. Neurol Med Chir (Tokyo). 2006;46(5):244-7.

11. Graves VB, Strother CM, Weir B, Duff TA. Vertebrobasilar junction aneurysms associated with fenestration: treatment with Guglielmi detachable coils. AJNR Am J Neuroradiol. 1996;17(1):35-40.

12. Uda K, Murayama Y, Gobin YP, Duckwiler GR, Viñuela F. Endovascular treatment of basilar artery trunk aneurysms with Guglielmi detachable coils: clinical experience with 41 aneurysms in 39 patients. J Neurosurg. 2001;95(4):624-32.

13. Yoon SM, Chun YI, Kwon Y, Kwun BD. Vertebrobasilar junction aneurysms associated with fenestration: experience of five cases treated with Guglielmi detachable coils. Surg Neurol. 2004;61(3):248-54.

\section{Endereço para correspondência} Johnathan de Sousa Parreira Rua Ildefonso dos Santos, 100, casa 23, Bairro Santa Clara 86036-590 - Londrina, PR, Brasil Telefones: (43) 3301-6497/(43) 9118-1185

E-mail: jsparreira@yahoo.com.br 